\title{
Etiology and Pathogenesis of Pericarditis from the Perspective of Traditional Chinese and Western Medicine
}

\author{
Fanbo Li, Gang Zheng* \\ Shaanxi University of Chinese Medicine, Xianyang 712046, Shaanxi Province, China \\ *Corresponding author: Gang Zheng, zhenggang2283@163.com
}

\begin{abstract}
Pericarditis is a common clinical pericardial disease with complex etiology, pathogenesis, and high recurrence rate. Interleukin-1 is an important inflammatory cytokine, which regulates the occurrence and development of inflammation by regulating IL- $1 \alpha$, IL-1 $\beta$, and IL-1RA; it plays an important role in pericarditis. In traditional Chinese medicine, the modern understanding of its etiology and pathogenesis has been improved. In this review, the etiology and pathogenesis of pericarditis are discussed from the perspective of traditional Chinese and Western medicine.
\end{abstract}

Keywords: Pericarditis; Combination of traditional Chinese and Western medicine; Pathogeny; Pathogenesis

Publication date: September 2021; Online publication: September 30, 2021

\section{Introduction}

Pericarditis refers to the inflammation of the pericardium. It is a common clinical pericardial disease. It is clinically divided into acute, persistent, recurrent, or chronic. There is no exact clinical data for the incidence rate and prevalence rage of pericarditis in China. The incidence rate of acute pericarditis in the northern region of Italy is 27.7 cases of acute pericarditis per 100 thousand people per year ${ }^{[1]}$.

\section{Etiology}

Western medicine believes that the etiology of pericarditis is very complex. Affected by regional and economic development, it can be divided into infectious, non-infectious, and idiopathic. Infectious factors include viruses, bacteria, fungi, and mycoplasma, among which the common viruses are Coxsackie virus and adenovirus. Under existing medical conditions, pericarditis caused by bacteria, fungi, and mycoplasma is rare due to the wide use of antibiotics; however, tuberculosis is the main cause of pericarditis in SubSaharan Africa ${ }^{[2]}$. Non-infectious factors, including tumor, post-cardiac injury syndrome, autoimmune diseases, metabolic syndromes, and some drugs, can lead to pericarditis. In Western Europe and North America, about $80 \%$ to $90 \%$ of cases are diagnosed as idiopathic ${ }^{[3,4]}$. The statistical results of hospitals in large cities in the United States also confirmed that the main cause of pericarditis is idiopathic ${ }^{[5]}$. In a retrospective analysis of 7,569 adult patients with pericarditis, tuberculosis was the main cause of pericarditis in developing countries, but China accounted for less than $5 \%$ of the cases in developed countries ${ }^{[6]}$. The etiology of recurrent pericarditis (RP) may be immune-mediated ${ }^{[7]}$.

\section{Pathogenesis}

At present, it is understood that pericarditis is closely related to the autoimmune mechanism of the innate 
immune system and adaptive immune system ${ }^{[8]}$, especially in idiopathic recurrent pericarditis without clear etiology. Moreover, the two immune systems can overlap and influence each other. Several RP patients are characterized by a moderate increase of inflammatory markers, presence of autoantibodies in serum, and associated autoimmune characteristics, clearly indicating that the disease is highly related to autoimmune diseases ${ }^{[8]}$. However, in some patients, it is marked by symptomatic serositis and fever as well as a significant increase of inflammatory parameters during an acute attack. There is a significant remarkable response against interleukin (IL), and the symptoms are completely relieved after anti-IL-1 treatment. This indicates that self-inflammation is an important pathogenesis ${ }^{[9]}$. In the IL-1 family, interleukin-1 $\alpha$, membrane-bound interleukin- $1 \alpha$ precursors, and interleukin- $1 \alpha$ precursors have complete biological activity ${ }^{[10]}$, and they can initiate interleukin- $1 \alpha$-interleukin-1R1 mediated signaling. In addition, interleukin- $1 \alpha$ precursors are cleaved by cal proteinase to produce IL- $1 \alpha-\mathrm{NTP}$ and the mature C-terminal form of IL- $1 \alpha$. When cells are necrotic, interleukin-1 $\alpha$ leaves the nucleus and appears in the cytoplasm. With its release, interleukin-1 $\alpha$ binds to interleukin-1R1 on adjacent living cells, causing inflammation. Conversely, apoptosis causes preinterleukin- $1 \alpha$ to migrate from the cytoplasm to the nucleus and bind tightly to chromatin, limiting interleukin- $1 \alpha$-mediated inflammation ${ }^{[11]}$. IL-1R2 is also present in the cytoplasm, where it binds to pre-IL- $1 \alpha$, preventing its cleavage and activation, thus playing a role in inhibiting or reducing inflammation ${ }^{[12]}$. The production of ${ }^{2 L-1} \beta$ can be divided into pathogen-associated molecular pattern molecules (PAMPs) and damage-associated molecular pattern molecules (DAMPs) ${ }^{[13]}$. The former triggers an inflammatory response by activating related pattern recognition receptors (PRRs) expressed in both immune and non-immune cells through microbial structures. The latter are components released or degraded by cells or extracellular matrix when cells or tissues are damaged, which can also trigger inflammation in the case of pathogens. When PAMPs and/or DAMPs are released, TLRs (toll-like receptors) are activated and NF-kB (nuclear factor kappa B) is translocated into the nucleus, promoting transcription and translation of inflammatory genes and the generation of bioactive IL- $1 \beta$ precursors ${ }^{[14]}$. IL- $1 \beta$ precursors are cleaved by caspase- 1 to produce biologically active mature IL- $1 \beta$, which is released from cells. However, cell death is not necessary for the release of active IL- $1 \beta$ under the action of caspase$1^{[15]}$. IL-1R1 can play a role in transmitting activation signals; IL-1 receptor antagonist (IL-1RA) binds closely to IL-1R 1 and blocks IL- $1 \alpha$ as well as IL- $1 \beta$ from binding to their corresponding receptors, thereby blocking IL-1 from producing inflammation.

\section{Understanding of pericarditis in traditional Chinese medicine}

There is not much understanding of the etiology and pathogenesis of pericarditis in traditional Chinese medicine. This disease is categorized under chest arthralgia, suspended drinking, cough, and asthma in traditional Chinese medicine. The insufficiency of natural endowment and deficiency of healthy Qi are the internal causes of the disease, while the invasion of warm heat, evil toxin, and phlegm stasis are the external causes. The location of the disease is mainly in the heart. The pathogenesis is understood as the deficiency of spleen Qi and the invasion by rheumatic heat evil, accumulation of heat-toxins in the lung and stomach with Yang brightness full of heat, warming evil that injures Yin, intermingled phlegm and blood stasis, blocking the pericardium, collection of rheum evil internally suppressing heart Yang, etc. ${ }^{[16]}$.

In summary, the etiology of pericarditis is complex, and the pathogenesis is closely related to the mechanism of autoimmune inflammation, among which IL-1 is the key player. In traditional Chinese medicine, pericarditis is unheard in ancient times. However, in modern times, it is believed that the key to its pathogenesis is the abundance of positive deficiency and evil. The pathogenesis can be summarized as the invasion of rheumatic heat evil, accumulation of heat-toxins, warming evil to hurt Yin, water-rheum suppressing the heart, etc. TCM needs to further improve its theoretical system and clarify its understanding of the disease. 


\section{Disclosure statement}

The authors declare that there is no conflict of interest.

\section{References}

[1] Imazio M, Gaita F, 2017, Acute and Recurrent Pericarditis. Cardiol Clin, 35: 505-513.

[2] Mayosi BM, 2007, Contemporary Trends in the Epidemiology and Management of Cardiomyopathy and Pericarditis in Sub-Saharan Africa. Heart, 93: 1176-1183.

[3] Imazio M, Spodick DH, Brucato A, et al., 2010, Controversial Issues in the Management of Pericardial Diseases. Circulation, 121(7): 916-928

[4] LeWinter MM, 2014, Acute Pericarditis. N Engl J Med, 371(25): 2410-2416.

[5] Vecchie A, Chiabrando JG, Dell MS, 2020, Clinical Presentation and Outcomes of Acute Pericarditis in a Large Urban Hospital in the United States of America. Chest, 158: 2556-2557.

[6] Imazio M, Gaita F, LeWinter M, 2015, Evaluation and Treatment of Pericarditis: A Systematic Review. JAMA, 314: 1498-506.

[7] Imazio M, Brucato A, Derosa F, et al., 2009, Aetiological Diagnosis in Acute and Recurrent Pericarditis: When and How. J Cardiovasc Med (Hagerstown), 10: 217-230.

[8] Tombetti E, Giani T, Brucato A, et al., 2019, Recurrent Pericarditis in Children and Adolescents. Front Pediatr, 7: 419.

[9] Imazio M, Brucato A, Pluymaekers N, et al., 2016, Recurrent Pericarditis in Children and Adolescents: A Multicentre Cohort Study. J Cardiovasc Med (Hagerstown), 17(9): 707-712.

[10] Malik A, Kanneganti TD, 2018, Function and Regulation of IL-1 $\alpha$ in Inflammatory Diseases and Cancer. Immunological Reviews, 281(1): 124-137.

[11] Dinarello CA, 2018, Overview of the IL-1 Family in Innate Inflammation and Acquired Immunity. Immunological Reviews, 281(1): 8-27.

[12] Zheng Y, Humphry M, Maguire JJ, et al., 2013, Intracellular Interleukin-1 Receptor 2 Binding Prevents Cleavage and Activity of Interleukin-1 $\alpha$, Controlling Necrosis-Induced Sterile Inflammation. Immunity, 38: 285-295.

[13] Chen L, Deng H, Cui H, et al., 2017, Inflammatory Responses and Inflammation-Associated Diseases in Organs. Oncotarget, 9(6): 7204-7218.

[14] Kaneko N, Kurata M, Yamamoto T, et al., 2019, The Role of Interleukin-1 in General Pathology. Inflamm Regener, 39: 12.

[15] Conos S, Lawlor K, Vaux D, et al., 2016, Cell Death is Not Essential for Caspase-1-Mediated Interleukin-1 B Activation and Secretion. Cell Death Differ, 23: 1827-1838.

[16] Yan JL, Xu YQ, 1989, Overview of Traditional Chinese Medicine Treatment of Pericarditis. Liaoning Journal of Traditional Chinese Medicine, 013(003): 46-48. 